\section{Le trouble déficitaire de l'attention avec hyperactivité}

\section{La génétique}

est-elle impliquée?

François Gonon, David Cohen

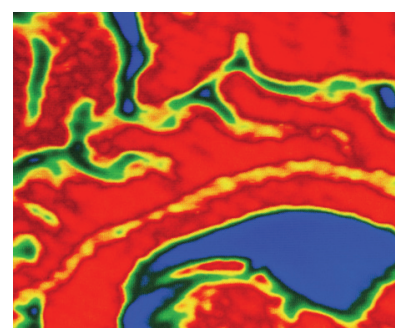

Le 30 septembre 2010, la revue The Lancet a publié une étude analysant le génome d'enfants souffrant du trouble déficitaire de l'attention avec hyperactivité (TDAH) [1]. Les auteurs ont observé des délétions et des duplications de portions du génome appelées variations du nombre de copies (en anglais: copy number variation, CNV). Les auteurs n'ont pris en considération que les CNV de grande taille (> $500 \mathrm{~kb}$ ). L'étude montre que ces CNV sont plus fréquents chez les enfants diagnostiqués TDAH que dans un groupe témoin et conclut que «le TDAH ne serait pas uniquement une construction sociale ». Cette conclusion a été reprise par plusieurs quotidiens français, y compris Le Monde du 2 octobre 2010. Pourtant, un examen critique s'impose.

L'étude portait sur 366 enfants chez lesquels le diagnostic de TDAH avait été porté et 1047 enfants non atteints. Parmi les enfants souffrant du TDAH, 33 avaient également un déficit intellectuel $(\mathrm{QI} \leq 70)$. Williams et al. ont observé que, parmi les 319 enfants souffrant du TDAH sans retard mental, 38 (soit $12 \%$ ) présentaient des CNV de grande taille. Ce type d'anomalies a également été observé chez 7,5\% des enfants témoins et chez 12 enfants souffrant de TDAH et de retard mental (soit $36 \%$ ). Si l'on considère ces derniers et le net écart de prévalence correspondant, on peut donc suivre les auteurs quand ils suggèrent que, pour un petit nombre d'enfants souffrant des deux pathologies, des anomalies génétiques pourraient avoir joué un rôle.

\author{
F. Gonon : Université \\ de Bordeaux \\ et CNRS UMR 5293 , \\ 146, rue Léo Saignat, \\ 33076 Bordeaux, France. \\ D. Cohen : service \\ de psychiatrie de l'enfant \\ et de l'adolescent, Université \\ Pierre et Marie Curie, APHP, \\ Pitié-Salpêtrière, \\ 75013 Paris, France. \\ francois.gonon@ \\ u-bordeaux2.fr
}

Plusieurs résultats de Groupehospitalier cette étude appellent un commentaire critique.

Premièrement, les auteurs conservent dans leur analyse les enfants avec retard mental. Or, par définition, le diagnostic de TDAH exclut la déficience intellectuelle. Si l'on ne considère, dans l'étude de Williams et al. , que les enfants souffrant du TDAH seul, l'écart de prévalence ( $12 \%$ versus $7,5 \%)$ est tout de même très modeste même s'il est significatif $(p=0,008)$. Notons qu'une autre étude de CNV n'a observé aucune différence significative entre enfants TDAH et enfants témoins [2]. Le Tableau I résume les méthodes et les principaux résultats de ces deux études. Deuxièmement, parmi les 38 CNV observés par Williams et al. chez les enfants TDAH sans retard mental, 17 correspondent à des locus déjà impliqués dans l'autisme ou la schizophrénie, mais même dans ce cas, le lien causal n'est pas établi. Par exemple, les CNV observés au niveau du locus $16 p 13.11$ ne concernent qu'une toute petite proportion de schizophrènes et d'enfants TDAH et ils sont aussi présents, quoique moins fréquemment, chez les témoins [1]. Et troisièmement, alors qu'une réplication sur la population islandaise est annoncée, les résultats ne sont que très partiellement présentés. On peut le regretter car les données cliniques dans cette cohorte sont de meilleure qualité et distinguent les sous-types de TDAH (mixte 


\section{Étude 1}

Williams et al. [1]

Rare chromosomal deletions and duplications in ADHD:

a genome wide analysis
Étude 2

Elia et al. [2]

Rare structural variants found in ADHD are preferentially

associated with neurodevelopmental genes

\begin{tabular}{|c|c|c|}
\hline $\begin{array}{c}\text { Taille } \\
\text { de l'échantillon } \\
(\mathrm{N})\end{array}$ & $\begin{array}{l}\text { Étude initiale } \\
\text { Royaume-Uni }\end{array}$ & Étude de réplication Islande \\
\hline TDAH & 366 & 825 \\
\hline Témoins & 1047 & 35243 \\
\hline $\begin{array}{l}\text { Protocole } \\
\text { de l'étude }\end{array}$ & $\begin{array}{l}\text { Cas-témoins } \\
\text { Cohortes caucc } \\
(1958) \text { et islan } \\
\text { RM non exclus } \\
\text { CNV considérés }\end{array}$ & $\begin{array}{l}\text { anglaise suivie dès la naissance } \\
\text { CODE) }\end{array}$ \\
\hline & Tests des CNV o & is l'autisme et la schizophrénie \\
\hline
\end{tabular}

Type de puce

Human660W-Quad BeadChip (TDAH), HumanHap550 BeadChip (témoins) (Illumina)

- Nombre et pourcentage d'enfants porteurs de CNV de grande taille :

TDAH total : 50 (14\%)

TDAH sans RM : 38 (12\%)

TDAH et RM : $12(36 \%)$

Témoins : 75 (7\%)

Principaux résultats

17 des 38 CNV observés chez les TDAH sans RM étaient localisés au niveau de locus impliqués dans l'autisme et la SCZ

- Réplication décrite mais ensemble des résultats non présenté pour la population islandaise

- Fréquence plus élevée de duplications 16p13.1 et des CNV associés à la $S C Z$, mais pas à l'autisme
335

2026

Trios TDAH-témoins

Cohorte d'ancêtres européens de la région de Philadelphie

Critères d'exclusion : prématurité, RM, autisme et TSA, psychoses, troubles majeurs de l'humeur, troubles neurodéveloppementaux, etc.

Détection de l'ensemble des CNV

Intérêts pour les gènes associés aux troubles neurodéveloppementaux : autisme, schizophrénie, syndrome de Tourette

Illumina Infinium II HumanHap550 BeadChip (Illumina)

- Nombre moyen de CNV par enfant :

TDAH : 27,4

Témoins : 26,9

- Pas de différences pour les délétions, les duplications, ni pour la taille des CNV entre cas et témoins

- Parmi les 222 CNV de grande taille, rares, hérités et observés chez 154 enfants TDAH, 28 étaient localisés au niveau de gènes impliqués dans des troubles neurodéveloppementaux connus (autisme, SCZ, mais aussi RM, troubles des apprentissages, Tourette, syndrome des jambes sans repos)

- Cette localisation spécifique est statistiquement plus fréquente dans cette population de patients TDAH

Tableau I. Un point de vue comparatif de deux études récentes sur les CNV associés au trouble de l'attention avec hyperactivité. TDAH : trouble déficitaire de l'attention avec hyperactivité ; SCZ : schizophrénie ; CNV : copy number variation ; RM : retard mental.

versus hyperactif/impulsif versus attentionnel). Or, dans des cohortes de cette importance, les troubles attentionnels peuvent être confondus avec des troubles visuospatiaux qui sont très fréquents dans les troubles développementaux associés à certains CNV, comme la microdélétion $22 q 11$ ou les duplications $15 q 11-q 13$, toutes deux associées à l'autisme ou à la schizophrénie [3].

En somme, contrairement à ce que suggèrent les auteurs, cette étude ne prouve nullement un lien causal entre CNV et TDAH. Pour renforcer leur point de vue, Williams et al. rappellent dans l'introduction et la discussion que l'héritabilité du TDAH est de $76 \%$. Par là même ils entretiennent l'idée qu'une héritabilité élevée implique nécessairement une cause génétique. La fausseté de cette idée a été bien soulignée par deux revues récentes [4] dont une consacrée au TDAH [5]. On rappellera seulement ici que l'héritabilité de la tuberculose est également de $70-80 \%$ et que 
les études d'héritabilité ne peuvent pas faire la distinction entre purs effets de gènes et interactions entre gènes et environnements.

Pour mettre en valeur leurs résultats, les auteurs rappellent dans l'introduction que les approches génétiques plus classiques, soit du génome entier, soit des gènes d'intérêt, n'ont pas permis d'identifier de manière certaine des gènes de susceptibilité pour le TDAH. Si l'on ne peut qu'être d'accord avec ce constat, il faut tout de même rappeler que les études initiales de gènes d'intérêt avaient rapporté des résultats très encourageants. Par exemple en 1996, la première étude portant sur l'association entre le TDAH et un polymorphisme du gène codant pour le récepteur D4 de la dopamine avait rapporté une prévalence de l'allèle $7 \mathrm{R}$ de $49 \%$ chez les patients TDAH et de $21 \%$ chez les témoins [6]. Les études ultérieures ont considérablement réduit cet écart (23\% versus $17 \%$ ) [7]. Comme l'a expliqué lonnadis, la première étude biomédicale qui utilise une nouvelle approche rapporte souvent des résultats plus spectaculaires que les suivantes [8]. Il est probable que l'étude de Williams et al. n'échappe pas à cette règle et il n'est donc nullement certain que son écart de prévalence ( $12 \%$ versus 7,5\%) demeure statistiquement significatif dans les études à venir. Ce qui pose problème pour l'information des nonspécialistes, c'est que les études initiales spectaculaires, comme celle de Williams et al., sont souvent publiées dans des journaux à fort facteur d'impact, et par conséquent plus souvent reprises par les médias. Les études ultérieures, qui viennent les tempérer ou même les contredire, ne sont publiées que par des revues scientifiques de second rang et ne sont donc pas médiatisées.

Williams et al. concluent que «le TDAH n'est pas uniquement une construction sociale». Pourtant leur étude ne permet pas d'entrevoir la mise au point d'un test génétique pour confirmer le diagnostic de TDAH. En l'absence de marqueur biologique, ce diagnostic repose sur une évaluation des symptômes, ce qui explique que la reconnaissance du TDAH présente une prévalence très différente d'un pays à l'autre. Le TDAH est donc bien actuellement une construction sociale $[9,10](\rightarrow)$.

$\rightarrow$ Voir l'article de J. Jupille, page 318 de ce numéro

Ceci ne veut pas dire que les patients qui souffrent de ce trouble n'ont pas besoin d'aide, y compris médicamenteuse [5]. Mais il nous paraît malsain, pour justifier un tel traitement, de mettre en avant une cause génétique du TDAH, qui n'est nullement démontrée et ne concernerait au mieux qu'un petit pourcentage de cas, alors que les facteurs de risque environnementaux du TDAH sont nombreux et avérés [5]. $\diamond$

Attention deficit hyperactivity disorder:

interpretation of a recent genetic study

\section{REMERCIEMENTS}

Nous remercions Claudine Laurent pour ses sug gestions. Ce travail a été soutenu par le Centre national de la recherche scientifique (CNRS UMR 5293), l'Université de Bordeaux et l'Université Pierre et Marie Curie, l'Agence nationale de la recherche (ANR-09-SSOC-006) ainsi que par l'Institut des sciences de la communication du CNRS (ISCC) et la Région Aquitaine.

\section{CONFLIT D'INTÉRÊTS}

Les auteurs déclarent n 'avoir aucun conflit d'intérêts concernant les données publiées dans cet article.

\section{RÉFÉRENCES}

1. Williams NM, Zaharieva I, Martin A, et al. Rare chromosomal deletions and duplications in attention-deficit hyperactivity disorder: a genomewide analysis. Lancet $2010 ; 376: 1401-8$.

2. Elia J, Gai X, Xie HM, et al. Rare structural variants found in attentiondeficit hyperactivity disorder are preferentially associated with neurodevelopmental genes. Mol Psychiatry 2010 ; 15 : 637-46.

3. Cohen D, Martel C, Wilson A, et al. Visual-spatial deficit in a 16-year-old girl with maternally derived duplication of proximal 15q. J Autism Dev Disord 2007 ; 37 : 1585-91.

4. Visscher PM, Hill WG, Wray NR. Heritability in the genomics era-concepts and misconceptions. Nat Rev Genet 2008 ; $9: 255-66$

5. Gonon F, Guilé JM, Cohen D. Le trouble déficitaire de l'attention avec hyperactivité : données récentes des neurosciences et de l'expérience nord-américaine. Neuropsychiatrie Enfance Adolescence 2010 ; $58: 273-81$.

6. LaHoste GJ, Swanson JM, Wigal SB, et al. Dopamine D4 receptor gene polymorphism is associated with attention deficit hyperactivity disorder. Mol Psychiatry 1996; 1: 121-4.

7. Gizer IR, Ficks C, Waldman ID. Candidate gene studies of ADHD: a metaanalytic review. Hum Genet 2009 ; $126: 51-90$.

8. loannidis JP. Why most published research findings are false. PLoS Med $2005 ; 2$ : el24.

9. Amaral OB. Psychiatric disorders as social constructs: ADHD as a case in point. Am J Psychiatry $2007 ; 164: 1612$.

10. Jupille J. Le trouble déficitaire de l'attention avec hyperactivité : le rôle des associations de parents. Med Sci (Paris) $2011 ; 27: 318-22$

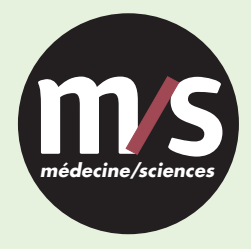

Tarifs d'abonnement M/S - 2011

Abonnez-vous

à Médecine/Sciences
> Depuis 20 ans, grâce à $\mathrm{m} / \mathrm{s}$, vous vivez en direct les progrès des sciences biologiques et médicales

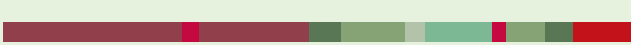

Bulletin d'abonnement page 253 dans ce numéro de $\mathrm{m} / \mathrm{s}$
TIRÉS À PART

F. Gonon 